\title{
Silencing and transcriptional properties of the imprinted Airn ncRNA are independent of the endogenous promoter
}

\section{Stefan H Stricker ${ }^{1,2}$, Laura Steenpass ${ }^{1}$, Florian M Pauler ${ }^{1}$, Federica Santoro, Paulina A Latos ${ }^{2}$, Ru Huang, Martha V Koerner, Mathew A Sloane ${ }^{3}$, Katarzyna E Warczok and Denise P Barlow*}

CeMM, Research Center for Molecular Medicine of the Austrian Academy of Sciences, Vienna, Austria

The Airn macro ncRNA is the master regulator of imprinted expression in the Igf $2 r$ imprinted gene cluster where it silences three flanking genes in cis. Airn transcription shows unusual features normally viewed as promoter specific, such as impaired post-transcriptional processing and a macro size. The Airn transcript is $108 \mathrm{~kb}$ long, predominantly unspliced and nuclear localized, with only a minority being variably spliced and exported. Here, we show by deletion of the Airn ncRNA promoter and replacement with a constitutive strong or weak promoter that splicing suppression and termination, as well as silencing activity, are maintained by strong Airn expression from an exogenous promoter. This indicates that all functional regions are located within the Airn transcript. DNA methylation of the maternal imprint control element (ICE) restricts Airn expression to the paternal allele and we also show that a strong active promoter is required to maintain the unmethylated state of the paternal ICE. Thus, Airn expression not only induces silencing of flanking mRNA genes but also protects the paternal copy of the ICE from de novo methylation.

The EMBO Journal (2008) 27, 3116-3128. doi:10.1038/ emboj.2008.239; Published online 13 November 2008 Subject Categories: chromatin \& transcription; RNA Keywords: Airn (Air); epigenetics; genomic imprinting; ncRNA; RNA splicing

\footnotetext{
*Corresponding author. CeMM, Research Center for Molecular Medicine of the Austrian Academy of Sciences, c/o Dr Bohr-Gasse 9/4, Vienna Biocenter Campus, 1030 Vienna, Austria. Tel.: +431 427754 610; Fax: + 431427795 46; E-mail: denise.barlow@univie.ac.at ${ }^{1}$ These authors contributed equally to this work

${ }^{2}$ Present address: Wellcome Trust Centre for Stem Cell Research, University of Cambridge, Cambridge CB2 1QR, UK

${ }^{3}$ Present address: CeMM c/o, AKH, Leitstelle 5H.J2.09, Währinger Gürtel 18-20, 1090 Vienna, Austria
}

Received: 18 April 2008; accepted: 17 October 2008; published online: 13 November 2008

\section{Introduction}

The classical view of gene regulation has been challenged in recent years by genome-wide studies that simultaneously investigated whole cell transcriptomes (Carninci et al, 2005; Cheng et al, 2005). The most surprising result of these studies was the high proportion of long non-protein coding transcripts (macro ncRNAs). Indeed, non-coding transcription units, excluding those regulating mRNA processing and translation, outnumber coding transcription units. The abundance and specific regulation of ncRNAs have been taken as an indication that many may have functional roles, most likely in the regulation of flanking genes. Most members of this new class of ncRNA are transcribed by RNAPII but they can show unusual transcriptional properties compared with mRNAs as they are often antisense to coding transcripts and enriched in the nuclear, non-polyadenylated and unspliced fraction (Mattick, 2005; Kapranov et al, 2007; Yasuda and Hayashizaki, 2008).

For a small number of macro ncRNAs, a functional role in gene silencing has been shown. These examples include Xist, the macro ncRNA required for $\mathrm{X}$ chromosome inactivation in female mammalian cells, Airn the repressive macro ncRNA of the Igf2r imprinted gene cluster (formerly known as Air but now renamed Airn by the HUGO Gene Nomenclature Committee), and Kcnq1ot1 the repressive macro ncRNA of the Kcnq1 imprinted gene cluster (Penny et al, 1996; Sleutels et al, 2002; Mancini-Dinardo et al, 2006). We focus here on the Igf $2 r$ imprinted gene cluster in which paternal-specific expression of the Airn ncRNA silences three genes in cis spread over a $300-\mathrm{kb}$ region. The Airn ncRNA promoter lies in an antisense orientation in $\operatorname{Ig} f 2 r$ intron 2 and drives a $108 \mathrm{~kb}$ mainly unspliced ncRNA that overlaps the Igf $2 r$ promoter; however, the two other silenced genes (Slc22a 2 and Slc22a3) that lie $80-150 \mathrm{~kb}$ upstream are not overlapped, nor do they share sequence homology with the Airn ncRNA (Pauler et al, 2007).

The Airn ncRNA is maternally repressed by a DNA methylation imprint that is set in oocytes on a CpG island that is part of a $3.65-\mathrm{kb}$ region genetically defined as the imprint control element (ICE) for the Igf $2 r$ imprinted cluster (Stoger et al, 1993; Wutz et al, 2001; Seidl et al, 2006). The Airn ncRNA promoter lies on the immediate $5^{\prime}$ side of the CpG island, thus all Airn ncRNA transcripts run through the CpG island (Figure 1A). The identical $3.65-\mathrm{kb}$ region on the paternal chromosome is not methylated during spermatogenesis and also remains unmethylated in diploid embryonic cells, thus the Airn ncRNA promoter is only active on the paternal chromosome. It is unknown why the ICE is unmethylated in sperm and diploid embryos, as both these stages experience waves of de novo DNA methylation ( $\mathrm{Li}$, 2002). The unmethylated state of the paternal ICE in embryonic stages may be a passive process that results from the 
A

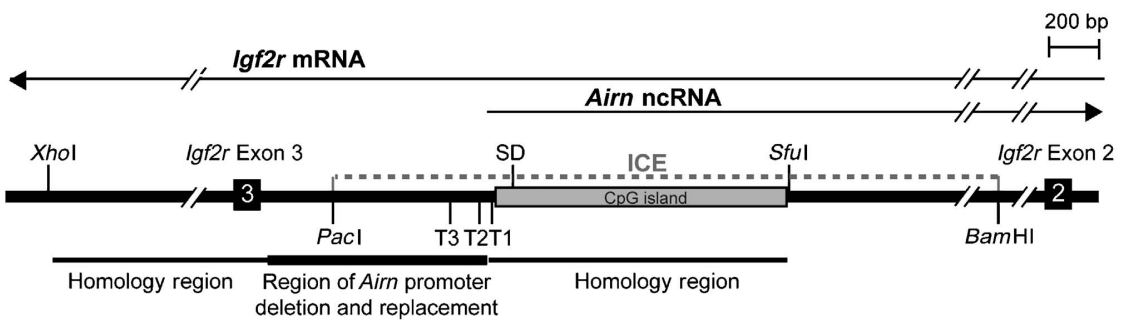

B

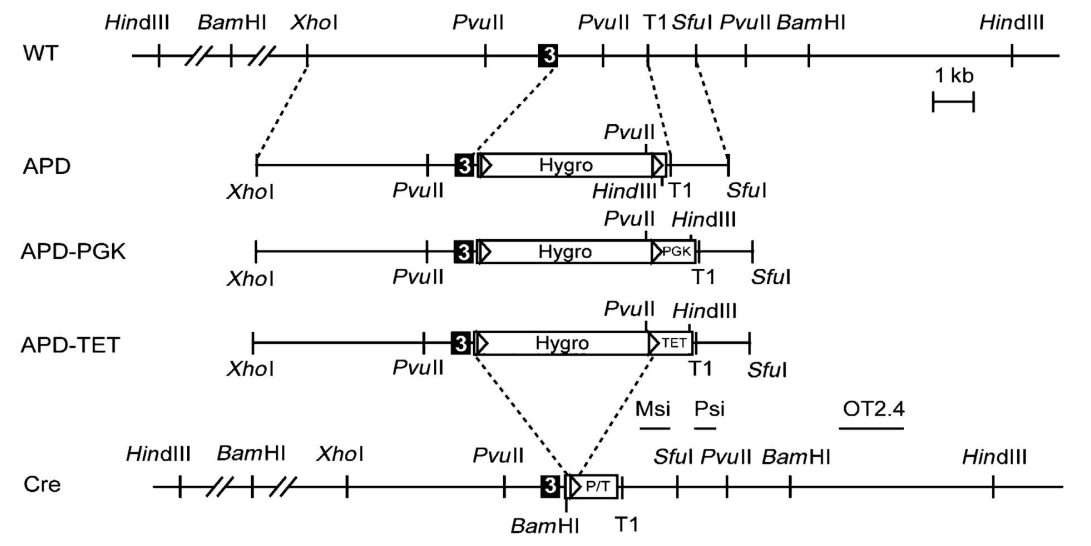

C

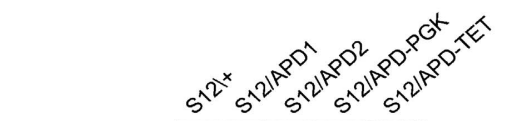

Digest: HindIII $\leftarrow$ - het
Probe: OT2.4

\section{E Digest: Pvull \\ Digest: Pvull}

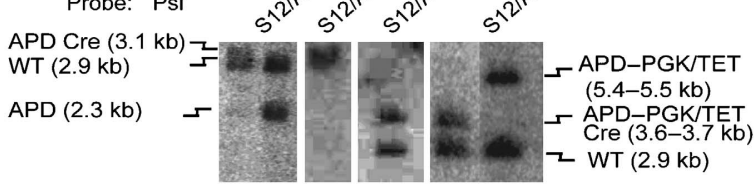

D

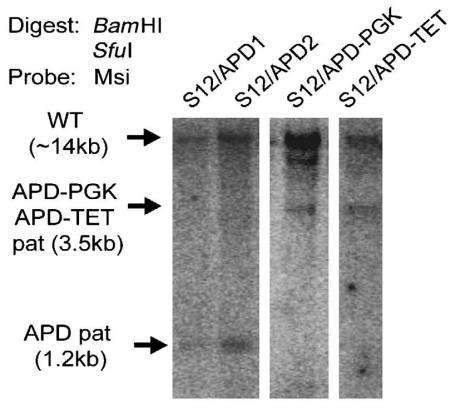

$\mathbf{F}$
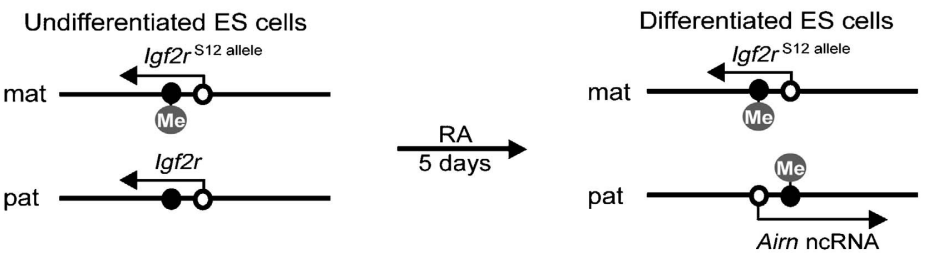

Figure 1 Deletion and replacement of Airn promoter constructs. (A) The Airn promoter lies in an antisense orientation in Igf2r intron 2. A 959bp fragment is deleted in the APD, APD-PGK and APD-TET alleles (thick line). The main transcription start site (T1) and downstream CpG island are unchanged. SD: Airn splice donor. A 3.65-bp PacI-BamHI fragment that has been genetically defined as the imprint control element (ICE) for the Igf2r imprinted cluster is indicated by the dotted line (Wutz et al, 2001). (B) Three constructs were used for targeting the Airn promoter deletion (APD) and the two promoter replacement alleles containing a PGK promoter (APD-PGK) or a TET promoter (APD-TET). Msi, Psi, OT2.4: Southern blot probes; hygro: hygromycin cassette. (C) Southern blot showing homologous targeting indicated by a 7.4-kb band. (D) Southern blot using the SfuI methyl-sensitive enzyme shows paternal targeting indicated by a $1.2 \mathrm{~kb}$ (APD) or 3.5 kb (APD-PGK, APD-TET) band. (E) Southern blot shows successful Cre recombination by loss of the unrecombined allele (APD 2.3 kb, APD-PGK 5.5 kb and APD-TET $5.4 \mathrm{~kb}$ ) and gain of the recombined allele (APD $3.1 \mathrm{~kb}$, APD-PGK $3.7 \mathrm{~kb}$, APD-TET $3.6 \mathrm{~kb}$ ). (F) Undifferentiated ES cells show biallelic expression of Igf2r and no expression of Airn. The Airn CpG island is methylated (Me) on the maternal allele (mat). Differentiation with retinoic acid (RA) leads to Airn upregulation, repression of Igf2r and methylation of the Igf2r CpG island on the paternal allele (pat) (PAL, manuscript submitted).

failure to attract the de novo methylation machinery in the paternal germ line and the availability only of maintenance methylation enzymes in embryonic stages, which only copy existing methylation patterns. However, it has been reported that short sequences in the paternal ICE actively protect it from de novo DNA methylation (Birger et al, 1999). More recently, it has also been suggested that active transcription might protect $\mathrm{CpG}$ island promoters from de novo DNA methylation. This suggestion arose from observations that weakening of promoters by mutating transcription factorbinding sites leads to promoter DNA methylation (Brandeis et al, 1994; Macleod et al, 1994).

The majority ( $>95 \%$ ) of transcripts from the Airn ncRNA promoter are unspliced and we have suggested that splicing suppression is the key feature of the Airn ncRNA, as other transcriptional properties such as its macro size and nuclear 
localization could depend on this (Seidl et al, 2006). In support of this model, retention at the site of transcription has been shown to be a consequence of impaired splicing and $3^{\prime}$ end processing in a human beta globin transgene (Custodio et al, 1999). Splicing and $3^{\prime}$ end processing leading to transcript termination are thought to occur co-transcriptionally (Kornblihtt et al, 2004; Bentley, 2005). This indicates that the Airn ncRNA promoter itself should regulate its transcriptional properties and thus its ability to silence genes in the Igf $2 r$ gene cluster. Here, we show by deletion and replacement of the Airn ncRNA promoter in an in vitro ES cell imprinting model that Airn ncRNA transcriptional properties, as well as Igf2 $r$ silencing, are independent of the endogenous Airn ncRNA promoter. All attributes of imprinted silencing, including DNA methylation and transcriptional silencing of the overlapped paternal Igf $2 r$ promoter, can be induced by Airn ncRNA expression driven by a strong exogenous promoter. However, a low expression level of the Airn ncRNA driven by a weak exogenous promoter is not sufficient for Igf2r silencing. Splicing suppression and termination are unchanged in the Airn promoter replacement allele, indicating that functional regions of the Airn ncRNA are located within its transcribed gene body not in its promoter. Surprisingly, ES cells lacking an Airn ncRNA promoter or containing a weakly expressed promoter gain DNA methylation of the paternal ICE. This indicates that the unmethylated state of the paternal ICE requires transcriptional run-through for protection from de novo methylation.

\section{Results}

\section{Establishment of Airn promoter deletion and promoter replacement by ES cell targeting}

Three targeting constructs were produced to modify the Airn ncRNA promoter that lies in an antisense orientation in Igf2r intron 2, close to exon 3 (Figure 1). Construct 1 generated a deletion (named APD: Airn promoter deletion) of the Airn ncRNA promoter that spans $959 \mathrm{bp}$, starting $3 \mathrm{bp}$ upstream of the $3^{\prime}$ splice acceptor site of Igf $2 r$ exon 3 and ending 1 bp upstream of the Airn main transcriptional start site (T1) (126236-127195 bp in accession number AJ249895). Constructs 2 and 3 were used to insert the ubiquitously expressed mouse phosphoglycerate kinase promoter (APDPGK) or a tetracycline-inducible promoter (APD-TET) into the APD in the same orientation as the endogenous Airn promoter.

The APD, APD-PGK and APD-TET targeting constructs were electroporated into D3 ES cells previously modified on the maternal allele to carry a single nucleotide polymorphism (SNP) in Igf2r exon 12 (PAL, manuscript submitted). This exon 12 modified allele is named S12, and ES cells carrying a maternally modified allele are labelled S12/ + (note that the maternal allele is written on the left side throughout the text). Southern blot analysis of 800 hygromycin-resistant colonies revealed homologous targeting for nine APD, six APD-PGK and eight APD-TET constructs (Figure 1C and data not shown). Except for one maternally targeted APD construct, all other homologous recombination events occurred on the paternal allele (Figure 1D and data not shown). A paternalspecific targeting bias in ES cells of unknown cause has been reported earlier for the $\operatorname{Igf} 2 r$ and Airn promoter regions (Wang et al, 1994; Sleutels et al, 2002, 2003). The selection cassette was removed by transient transfection with a Cre recombinase expression plasmid (Figure 1E). Four homologously targeted ES cell lines were used in this study: S12/ APD-1 and S12/APD-2 (two independent APD clones that lack a paternal copy of the Airn promoter), S12/APD-PGK (that replaces paternal copy of the endogenous Airn promoter with the ubiquitously expressed PGK promoter) and S12/APD-TET (that replaces paternal copy of the endogenous Airn promoter with an inducible TET promoter). The sequence of the APD-PGK and APD-TET replacement promoters was amplified from the targeted ES cell genomic DNA, which shows the correct targeting of the replacement promoters (Supplementary Figure 1). Note that in all these cells the maternal chromosome carries the introduced Igf $2 r$ exon 12 SNP and the paternal chromosome carries the Airn promoter modification.

\section{APD and replacements change Airn expression in ES cells}

Airn expression is undetectable in undifferentiated ES cells, which show biallelic Igf2 $r$ expression. Imprinted expression of Igf2 $r$ arises during ES differentiation coincident with paternal Airn ncRNA expression (Figure $1 \mathrm{~F}$ and PAL, manuscript submitted). Therefore, the four ES cell lines were differentiated by withdrawal of LIF and addition of retinoic acid (RA) to assay the expression of the Airn ncRNA. After 2 days, ES cultures gained visual signs of differentiation and were virtually free of undifferentiated cells after 5 days as assessed by phenotypic appearance.

To test whether the APD is sufficient to eliminate Airn expression, cDNA from different time points of ES cell differentiation was produced and expression was analysed by QPCR using a Taqman probe located $54 \mathrm{~kb}$ downstream of the Airn promoter (Figure 2A). Figure 2B shows that undifferentiated control ES cells $(\mathrm{S} 12 /+/ \mathrm{d} 0)$, as well as undifferentiated ES cells with a deletion of the paternal promoter (S12/APD2/d0), lack detectable Airn ncRNA expression. After differentiation for up to 14 days, control S12/ + ES cells showed strong Airn expression; however, differentiated ES cells with a deletion of the paternal promoter (S12/APD1/ d5 and S12/APD2/d5) showed only background Airn expression levels $(<1 \%$ of wild type; Figure $2 \mathrm{~B})$. The data show that the $959 \mathrm{bp}$ deleted region contains essential parts of the Airn promoter. These findings were confirmed by RNase Protection Assay, which showed a complete absence of Airn ncRNA in differentiated APD-1 ES cells using a probe located $25.8 \mathrm{~kb}$ downstream of the Airn promoter (data not shown).

Figure 2B shows Airn expression in S12/APD-PGK and S12/APD-TET cells in which the Airn promoter is replaced by the ubiquitously expressed PGK promoter or the non-induced TET promoter. The PGK promoter should be active in undifferentiated and differentiated ES cells, whereas the TET promoter is predicted to be silent in the absence of the transactivator. In undifferentiated ES cells, the APD-PGK allele expressed Airn at $\sim 50 \%$ of the level found in wildtype ES cells differentiated for 5 days. In ES cells differentiated for 5 days, the APD-PGK allele showed increased Airn expression equivalent to that seen in wild-type cells differentiated for a similar period; however, in ES cells differentiated for 14 days the APD-PGK allele produces significantly less Airn (Figure 2C). This shows that an exogenous PGK 
A
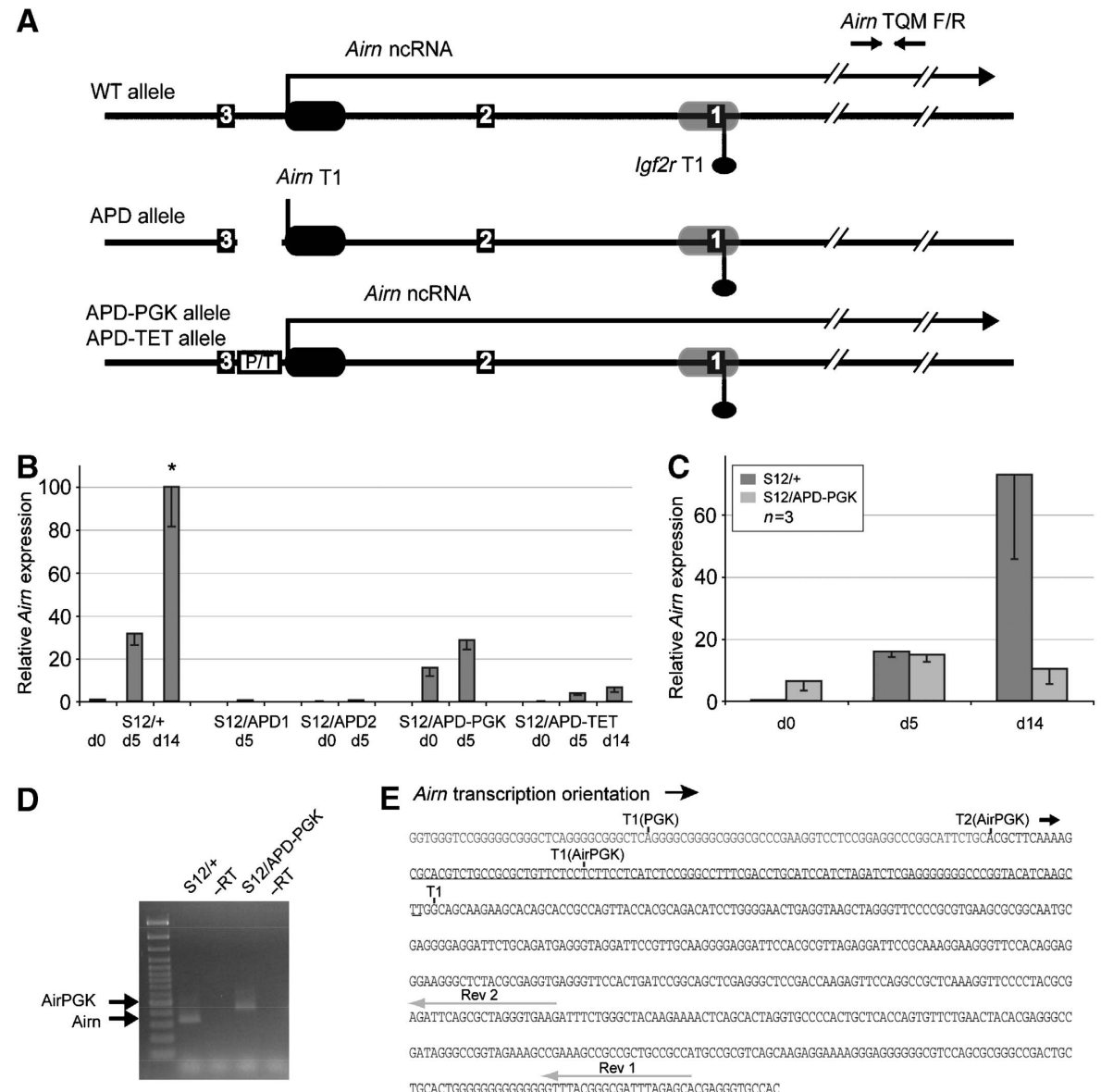

Figure 2 Airn expression changes in promoter deletion and replacement cells. (A) Map of the wild-type Airn promoter (WT), the Airn promoter deletion (APD) and replacement alleles (APD-PGK and APD-TET). Airn TQM F/R: QPCR assay used in (B, C). Numbered black boxes: Igf2r exons. Black oval: Airn CpG island, grey oval: Igf2r CpG island. The positions of the Airn and Igf2r transcription start sites with respect to the CpG island were determined by 5'RACE (Seidl et al, 2006). (B) QPCR showing Airn expression in targeted ES cells differentiated for 0, 5 or 14 days. Control undifferentiated ES cells $(\mathrm{S} 12 /+$ ) lack Airn expression (day 0). Differentiation for 5 or 14 days increases Airn expression strongly. S12/APD cells lack Airn expression at all time points. S12/APD-PGK cells show moderate Airn expression (50\% of 5 day control) in undifferentiated cells (d0) that increases to WT levels by day 5. S12/APD-TET cells show weak Airn expression under non-induced conditions but only in differentiated cells $\left(3.8 \%\right.$ at 5 days, $6.5 \%$ at 14 days relative to 14 day control). ${ }^{*}$ Set to 100 . Error bars represent standard deviation of three technical replicates (one biological replica). (C) QPCR comparing Airn expression in three biological replicates of S12/ + and S12/ APD-PGK ES cells, differentiated for 0, 5 or 14 days, shows equal Airn expression at 5 days in S12/ + and S12/APD-PGK that is reduced at 14 days to $15 \%$ of control levels. Details as in (B). (D) 5'RACE products to visualize Airn transcription starts in 14-day differentiated S12/ + and S12/PGK cells. (E) Sequence showing locations of published Airn (T1) and PGK (T1(PGK)) transcription start sites and two transcription start sites of PGK-driven Airn (T1(AirPGK) and T2(AirPGK)). Rev 1, Rev 2: RACE primers; underlined: PGK promoter sequence; black font: PGKAirn transcript; black arrow: transcript direction.

promoter can drive expression of the Airn ncRNA in undifferentiated ES cells and be upregulated during early differentiation to a similar level as the endogenous promoter. However, in contrast to the endogenous promoter, the PGK promoter is not further upregulated at later differentiation stages (Figure 2C). The non-induced APD-TET allele was not expressed in undifferentiated ES cells, but early and later differentiated ES cells expressed low amounts of Airn equivalent to $\sim 7 \%$ of the level seen in wild-type differentiated cells (Figure 2B). We next used $5^{\prime}$ RACE to map the transcription start site of the APD-PGK transcript using nested primers located downstream from the Airn T1 transcription start (Figure 2D and E). The APD-PGK $5^{\prime}$ RACE product was larger than the wild-type product and sequencing revealed that the PGK promoter did not use its standard transcription start but instead used two new transcription start sites that added 106 or $70 \mathrm{bp}$ to the endogenous Airn T1 transcription start site.

\section{Replacement of the Airn promoter does not abolish splicing suppression of the Airn transcript}

To test whether splicing suppression of the Airn ncRNA is regulated by its endogenous promoter, we quantified by QPCR the expression levels of the unspliced Airn and of the four known Airn splice variants (SV1, SV1a, SV2 and SV3) in wild-type $\mathrm{S} 12 /+$, APD, APD-PGK and APD-TET ES cells differentiated for 5 days (Figure $3 \mathrm{~A}$ and $\mathrm{B}$ ). The results show that APD cells carrying a paternal APD lack all Airn spliced variants, thus the spliced products share the same essential promoter elements as the unspliced product. The APD-PGK and APD-TET alleles produced all four spliced Airn variants in addition to unspliced Airn with a moderately changed ratio. The amount of unspliced Airn produced by the APD-PGK and APD-TET alleles was 98 and $12 \%$ of wildtype levels, respectively. Spliced variants SV1 and SV1a were decreased (APD-PGK: 42\% (SV1), 58\% (SV1a); APD-TET: 
A
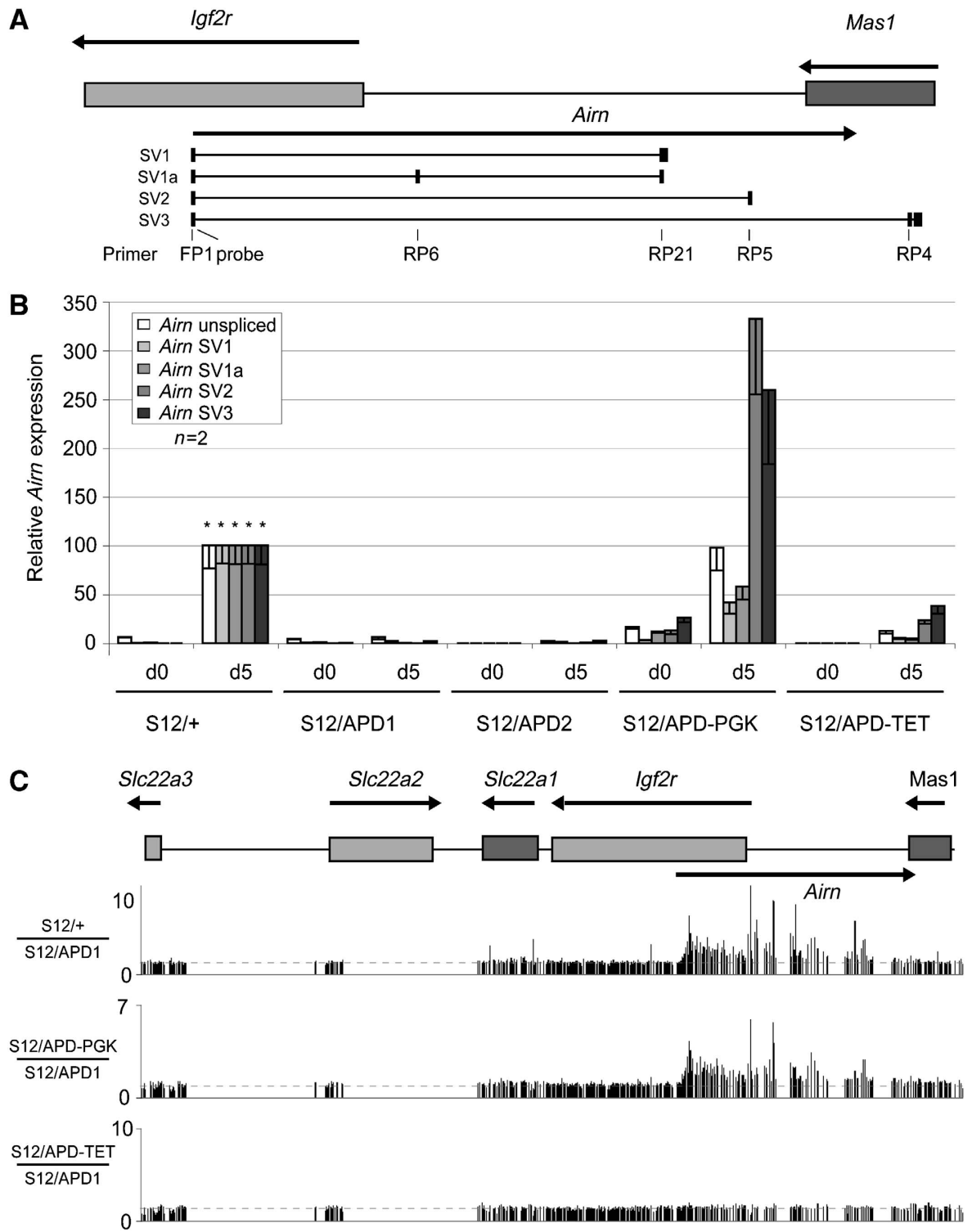

Figure 3 The PGK promoter expresses full-length Airn. (A) Map showing unspliced Airn (black arrow) and the known Airn splice variants and primers used in the splice variant-specific QPCR. The unspliced and spliced transcripts use the same transcription start sites and all four spliced variants use the same 53 bp exon 1. SV3 extends beyond the mapped end of the unspliced Airn transcript. A common forward primer (FP1) and Taqman probe (Probe) are combined with different reverse primers (RP21 (SV1), RP6 (SV1a), RP5 (SV2) and RP4 (SV3)). (B) Splice variantspecific QPCR. S12/ + control cells mostly lack expression of all splice variants at day 0 and show maximum expression at day 5. Spliced variants represent $<5 \%$ of total Airn transcripts (Seidl et al, 2006); however, for purposes of comparison with the targeted alleles, the expression levels of unspliced and splice variants transcripts in S12/+ cells at day 5 is set to $100(*)$. S12/APD cells and S12/APD-TET lack Airn splice variants at day 0, whereas S12/APD-PGK shows low expression levels at day 0. Expression of splice variants increases in S12/APDPGK and S12/APD-TET ES cells at day 5. Mean values and standard deviations of two biological replicates are shown. (C) cDNA hybridization experiment on a custom PCR genomic tiling array (Regha et al, 2007). Top: map of the Igf2 $r$ imprinted cluster contained on the tiling array (note that only the promoter regions of Slc22a2 and Slc22a3 were included on the array). The three lower tracks on the $y$ axis show RNA signal ratios of S12/ + , S12/APD-PGK, S12/APD-TET relative to S12/APD1 in 5 day differentiated cells. Outside the Airn transcription unit the mean ratio is close to 1 (dashed line), indicating equal expression in the experimental (S12/+, S12/APD-PGK and S12/APD-TET) and reference cell lines (S12/APD1). S12/ + cells show elevated levels inside the Airn transcription unit that is reproduced by S12/APD-PGK, indicating no significant gain of splicing or premature termination in this replacement allele. Weak Airn expression from S12/APD-TET was not detected.

$5 \%$ (SV1) and 5\%(SV1a)); however, spliced variants SV2 and SV3 were expressed at higher levels relative to unspliced Airn (APD-PGK: 332\% (SV2), $260 \%$ (SV3); APD-TET: $23 \%$ (SV2), $38 \%$ (SV3)). As it has been shown earlier that only a small minority of Airn ncRNA transcripts $(<5 \%)$ are spliced (Seidl et al, 2006), this 2- to 3-fold upregulation of two of the four splice variants in the Airn promoter replacement alleles does not indicate a significant loss of splicing suppression.
To gain a more complete view of the ratio of unspliced and spliced Airn transcripts, we used a custom genome tiling array prepared from PCR amplicons spanning the complete Igf2r/Airn region (Regha et al, 2007). In Figure 3C, the genome tiling array was hybridized with two cDNA populations, one labelled with Alexa 555 was prepared from S12/ APD differentiated ES cells that lack Airn expression, the other labelled with Alexa 647 was prepared from either 
S12/ + (top row), S12/APD-PGK (middle row) or S12/APDTET (bottom row) differentiated ES cells. The vertical bars in Figure 3C show signal intensity ratios between the cell lines. Regions showing similar expression in both cell lines (such as Slc22a1) have ratios close to one (dashed grey line). However, throughout the Airn transcription unit S12/ + and S12/APD-PGK show significantly higher signal ratios (75 and $93 \%$ of signals are elevated), the signal variance within Airn is related to probe lengths on the tiling array. The pattern of wild-type Airn transcription (top row) closely resembles that of PGK-Airn (middle row), indicating no major gain of splicing or premature termination in S12/APD-PGK cells. In S12/APD-TET cells, the lowly expressed TET-Airn transcript is not detected by the tiling array.

\section{Can Airn expressed from an exogenous promoter silence Igf2r?}

We have shown earlier that expression of Airn leads to silencing in cis of the paternal Igf2r promoter and gain of DNA methylation on the Igf2r promoter CpG island (Sleutels et al, 2002). To test whether Airn driven from an exogenous promoter will induce gain of DNA methylation on the Igf2r promoter, genomic DNA was extracted from undifferentiated and differentiated ES cells and the methylation status of several methyl-sensitive restriction enzyme sites was analysed by Southern blot (Figure 4 and data not shown). In wild-type S12/ + ES cells, DNA methylation of a NotI site, which is diagnostic of the methylation status of the Igf $2 r$ promoter CpG island (Stoger et al, 1993), was absent in

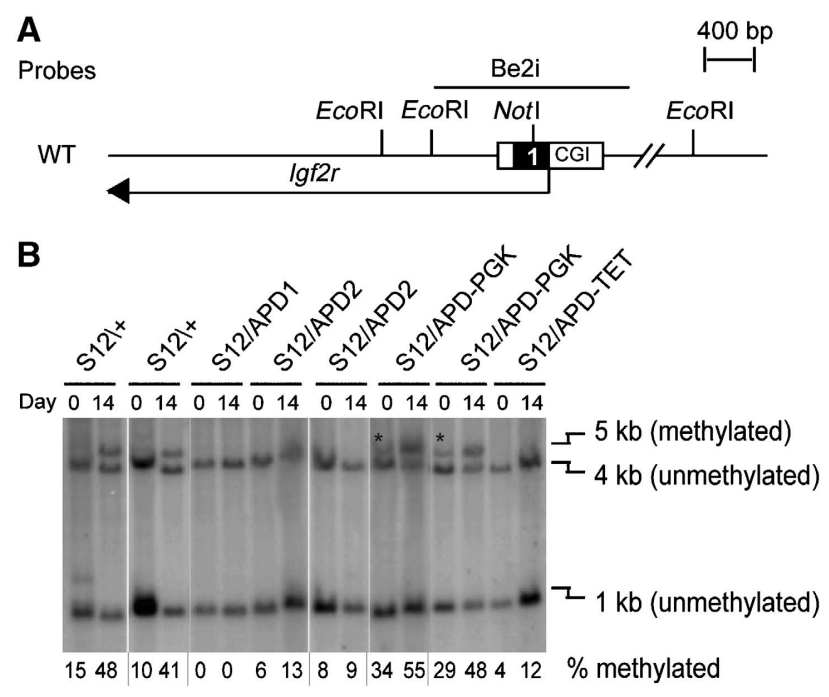

Figure 4 Igf2r promoter DNA methylation. (A) Map of an 8-kb region containing the Igf2r promoter (black arrow: transcription orientation). White box: CpG island; black box 1: Igf2r exon 1. Be2i: Southern blot probe. (B) Southern blot on genomic DNA of days 0 and 14 RA differentiated ES cells using EcoRI and the methylsensitive NotI enzyme and probe Be2i. One of two biological replicates is shown. S12/ + ES cells at day 14 show DNA methylation on NotI that is indicative of general methylation levels on the CpG island on the paternal allele (Stoger et al, 1993), whereas S12/ APD1 and S12/APD-TET lack methylation. S12/APD-PGK cells show partial NotI methylation at day 0 (29-34\%, asterisks) and full methylation at day $14(48-55 \%$, note that only the paternal allele is methylated (Stoger et al, 1993)). Numbers below indicate the relative intensities of methylated bands (ImageJ). undifferentiated cells and reached a level of almost $50 \%$ at day 14 , which indicates full methylation of the paternal allele in diploid cells. Differentiated APD1 and APD2 cells that lack the paternal Airn promoter as well as differentiated APD-TET cells with a replacement TET promoter, all failed to gain methylation of the $\operatorname{Ig} f 2 r$ promoter $\mathrm{CpG}$ island. In contrast, APD-PGK cells with the replacement PGK promoter gained normal levels of DNA methylation on the Igf2r promoter CpG island in differentiated cells. In addition, APD-PGK cells show a low level of Igf2r promoter DNA methylation in undifferentiated ES cells $\left(^{*}\right)$. These data indicate that high levels of Airn driven from the exogenous PGK promoter can silence Igf2r in cis, whereas low levels of Airn driven from an exogenous non-induced TET promoter cannot.

\section{PGK promoter-driven expression of Airn leads to transcriptional silencing of Igf2r}

RNA FISH (fluorescence in situ hybridization) using intronic probes allows visualization of nascent transcription on a single-cell level, and was used to investigate Igf2 $r$ imprinted expression. Figure 5A shows typical images using a strandspecific hybridization probe located in $\operatorname{Ig} f 2 r$ intron 1 where each fluorescent spot indicates nascent transcription of Igf $2 r$. Using intronic probes, only $32-47 \%$ of control differentiated $\mathrm{S} 12 /+$ cells show a fluorescent signal for Igf2r. The lack of fluorescent signal in every cell in the population likely arises from stochastic gene expression where at any one time point, only a proportion of nuclei are transcribing Igf $2 r$ even though all cells may contain the gene product.

Figure 5B (left panel) shows RNA FISH analysis using strand-specific Igf2r hybridization probes located either in intron 1 (FP1, left bar) or intron 5 (FP3, right bar). For each data set, at least 100 cells with a fluorescent signal were counted by visual inspection and the percentage of cells with no spot (0), a single spot (1), two spots (2) or multiple spots $(+)$ was determined. An independent counter with no knowledge of the cell genotype repeated the analysis and error bars indicate differences between these two counters. In wild-type S12/ + cells, 53-66\% of cells lack any fluorescent spot signal, $30-38 \%$ show a single spot and 3-7\% show double spots. Double spots represent biallelic expression of Igf $2 r$, thus the majority of S12/ + differentiated ES cells show monoallelic Igf $2 r$ expression. In S12/APD cells that have a paternal deletion of the Airn promoter, 47-49\% of cells lack any fluorescent spot signal, $22-24 \%$ show a single spot and 24-25\% show double spots. Thus, APD cells show an increase of approximately five-fold in the number of nuclei showing two spots, indicating biallelic expression of $\operatorname{Igf} 2 r$. This finding is confirmed by analysis of a second independent clone containing the APD (S12/APD1; Figure 5B, right panel). The similar numbers of nuclei with single spots in wild-type and APD cells was unexpected, and we interpret this as stochastic gene expression leading to an underestimation of the percentage of APD cells with double spots. In contrast to S12/APD cells, the APD-PGK cells in which the PGK promoter drives Airn expression showed a fluorescent spot signal distribution similar to the wild type (66-69\% of cells lack any fluorescent spot signal, 25-29\% show a single spot and $5 \%$ show double spots). These data indicate that Airn expressed from an exogenous PGK promoter is sufficient for transcriptional silencing of Igf2r. 

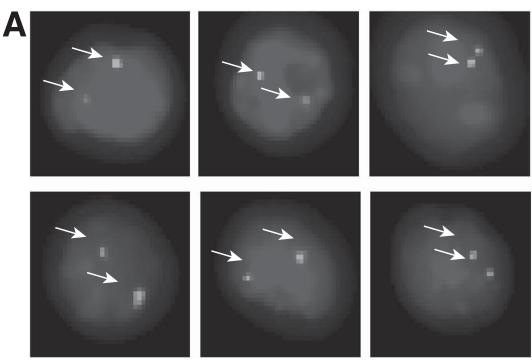

Double transcription spots
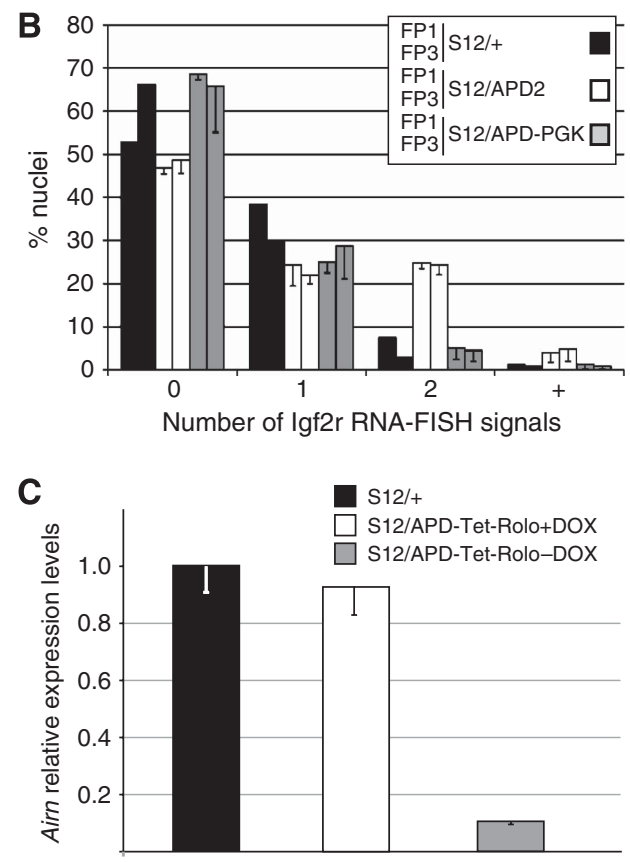
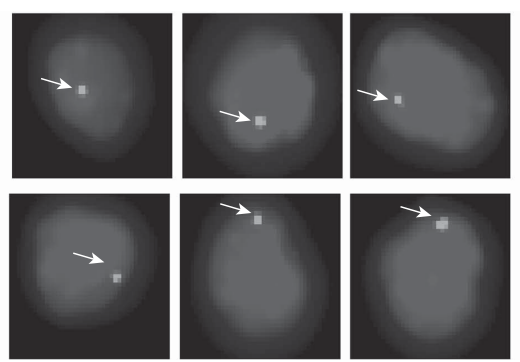

Single transcription spots
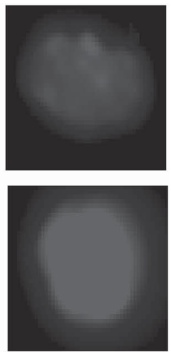

No spot
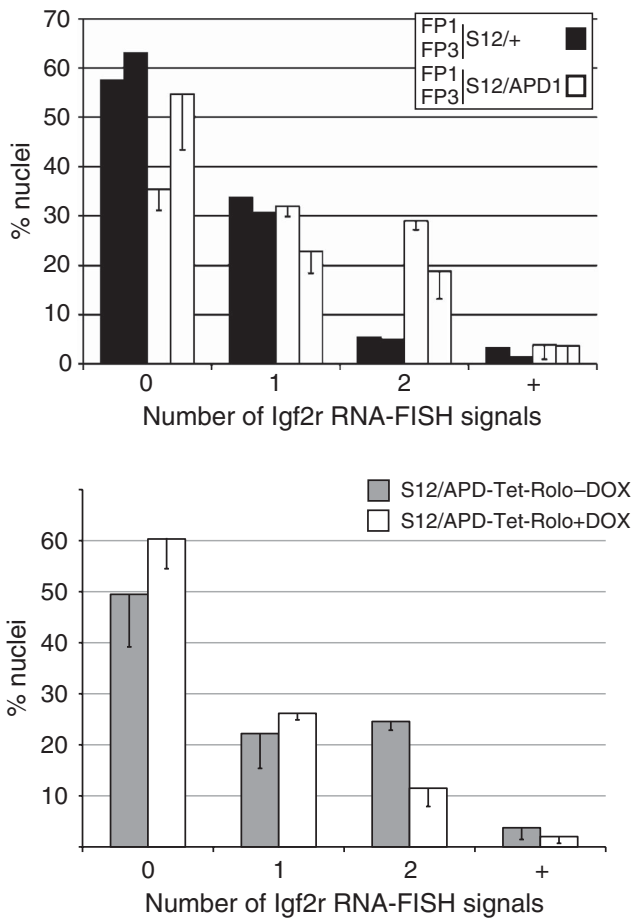

Figure 5 PGK-Airn and induced TET-Airn silence Igf2r in cis. (A) RNA FISH with the Igf2r intronic FP1 strand-specific probe in S12/APD ES cells. Representative examples of day 5 RA differentiated cells with double, single and no transcription spots are shown. (B) Left panel: quantification of Igf2r transcription by FISH using intronic probes FP1 (left bar) and FP3 (right bar). Double spots (2) indicate biallelic expression, single spots (1) monoallelic or stochastic biallelic expression. + : multiple spots indicate unspecific signals. Control S12/ + cells (dark grey bars) show mainly single spots. Airn promoter deletion S12/APD2 (white bars) shows an increase in cells with double spots, indicating a loss of imprinted expression. S12/APD-PGK (light grey bars) cells show mainly single spots consistent with imprinted Igf2r expression. Error bars represent means of two independent counts (one performed blind). Right panel: as left panel, but using an independently targeted APD allele (S12/APD1). (C) Left panel shows induced Airn expression levels assayed by QPCR as described in Figure 3 in APD-TET-Rolo ES clones carrying a transactivator (rtTA) gene targeted into the ROSA26 locus (Beard et al, 2006). Treatment of S12/APD-TET-Rolo cells with Doxycycline during 5d of ES cell differentiation leads to induction of the Airn ncRNA comparable to wild-type levels. Right panel shows an RNA FISH analysis of Igf2r transcription in S12/APD-TET-Rolo cells induced to express high levels of Airn. Uninduced S12/APD-TET-Rolo cells show a transcription pattern indistinguishable from S12/APD cells (double spots: 24\%, -Dox). Induction of Airn ncRNA expression decreases biallelic Igf2r expression (double spots: $12 \%$, + Dox). Error bars represent means of three independent counts (two were performed blind).

\section{High TET-Airn levels can induce Igf2r silencing}

The analysis of the APD-TET promoter in Figures 3B and 4B showed that this promoter replacement allele expressed low levels of Airn that were insufficient to induce DNA methylation of Igf $2 r$ in differentiated ES cells. In contrast, Figures 3B, $4 \mathrm{~B}$ and $5 \mathrm{~B}$ show the APD-PGK allele produced high levels of Airn that were sufficient for transcriptional silencing of Igf $2 r$. To test whether transcriptional activity or the promoter sequence of the replacement Airn promoter is related to its ability to silence Igf2 $r$, we induced expression of the APD-TET promoter. We targeted a tetracycline-inducible rtTA transactivator gene into the ubiquitously expressed ROSA26 locus of S12/APD-TET cells that allows rtTA expression to be driven by the ROSA26 promoter (Beard et al, 2006). The resultant cell line (S12/APD-TET-Rolo) was differentiated for 5 days in the presence of doxycycline and showed a nine-fold induction of Airn that is similar to wild-type levels at day 5 (Figure 5C, left panel). Figure 5C (right panel) shows an RNA FISH analysis of $\operatorname{Ig} f 2 r$ transcription in the same S12/APD-TETRolo cells analysed in Figure 5C (left panel). The results show that induction of APD-TET promoter causes a significant reduction of cells expressing Igf $2 r$ biallelically compared with the untreated control (26\% minus doxycycline and 12\% plus doxycycline). This reduction, although significant (unpaired t-test: $P<0.005)$, is less than that observed in Figure 5B with wild-type or APD-PGK promoters that showed 
only 5-7\% double spots. We therefore examined DNA methylation at an SfuI site that is diagnostic for the methylation status of the Airn CpG island (Stoger et al, 1993) in the APDTET-Rolo cells and found in contrast to the wild-type allele, that it was methylated in differentiated ES cells despite continuous doxycycline induction (see below and Figure 7). This indicates that only a sub-population of cells is responsible for producing high APD-TET expression following doxycycline treatment, thus the proportion of cells showing a loss of biallelic Igf $2 r$ is reduced. The gain of imprinted $\operatorname{Ig} f 2 r$ expression in induced APD-TET-Rolo cells was also accompanied by a slight gain of DNA methylation (data not shown) on the NotI site that is diagnostic for the methylation status of the Igf2r CpG island (Stoger et al, 1993). Thus, the data shows that induced expression of Airn from the APD-TET promoter is able to silence Igf $2 r$, indicating that the expression level of the Airn ncRNA is a key factor in Igf $2 r$ silencing.

\section{PGK-Airn silences the paternal Igf2r promoter}

The RNA FISH experiments described above demonstrated that PGK-driven Airn was sufficient to induce imprinted Igf $2 r$ expression. However, RNA FISH cannot identify the parental alleles in a diploid nucleus. We therefore used the SNP introduced into Igf2r exon 12 (named S12) to determine whether PGK-driven Airn was able to mimic the wild-type Airn ncRNA and specifically silence the paternal Igf2r promoter. Using an allele-specific QPCR assay (PAL, manuscript submitted), the expression status of the two parental Igf $2 r$ alleles was analysed in S12/APD, S12/APD-PGK and in noninduced S12/APD-TET differentiated ES cells. In Figure 6A, control undifferentiated wild-type $\mathrm{S} 12 /+$ ES cells were assigned a 1:1 ratio of maternal and paternal expression in agreement with earlier reports that Igf2r shows biallelic expression in undifferentiated ES cells (Wang et al, 1994). After 5 days, differentiated S12/ + cells show a maternal/ paternal ratio of 19:1, indicating that wild-type differentiated ES cells have gained imprinted maternal-specific Igf2 $r$ expression. A similar increased maternal/paternal ratio from 1.4:1 in undifferentiated cells to $16: 1$ in differentiated cells was seen in S12/APD-PGK differentiated cells, indicating maternal-specific Igf2r expression. Surprisingly, S12/APD and S12/ APD-TET differentiated cells also showed a similar increased maternal/paternal ratio in differentiated ES cells (from 1.8:1 to $14: 1$ and from $1.4: 1$ to $14: 1$ ). This result is surprising because it contradicts the RNA FISH and the DNA methylation analyses described above, which showed that the APD and non-induced APD-TET alleles did not silence the paternal Igf2 $r$ promoter. To explain how the paternal Igf2 $r$ allele appears to lack transcription silencing yet fails to produce a stable transcript that can be quantified by QPCR, we considered that the Airn promoter $959 \mathrm{bp}$ deletion may have disturbed the splice acceptor of Igf2r exon 3 specifically in differentiated ES cells. We performed a non-quantitative PCR using primers spanning Igf $2 r$ exons $2-4$ on cDNA from differentiated S12/APD cells and identified mis-splicing on the APD allele from exons 2 to 4 (data not shown), which would introduce multiple premature stop codons in the first $500 \mathrm{bp}$ of $I g f 2 r$ and be predicted to reduce transcript stability through nonsense-mediated RNA decay (NMD).

As NMD requires active translation, we treated ES cells differentiated for 5 days with the translation inhibitor emetine. Figure $6 \mathrm{~B}$ shows that the maternal/paternal Igf2r ratio in
A

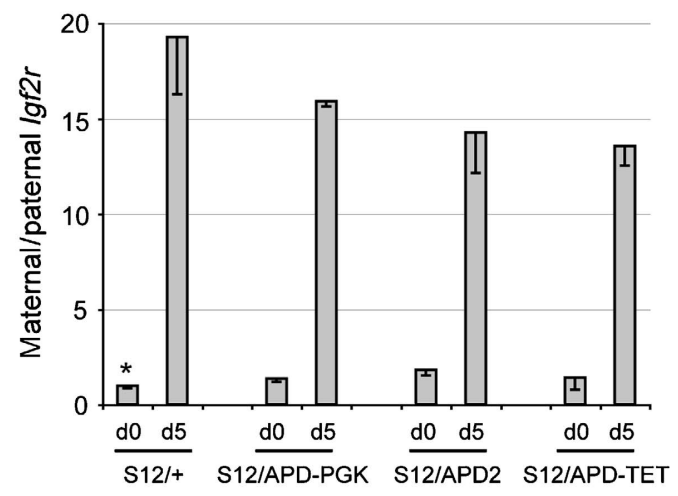

B $n=3 \quad P>0.1 \quad P<0.002 \quad P<0.002 \quad P<0.002$
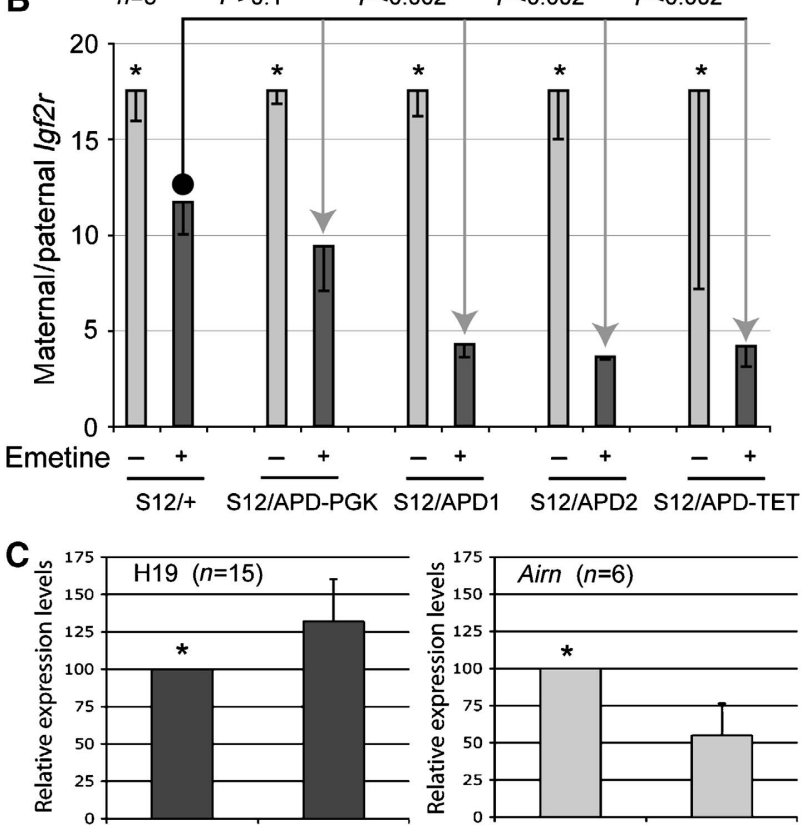

Emetine

Figure 6 PGK promoter-driven Airn silences paternal Igf2r. (A) Allele-specific QPCR showing the ratio of maternal/paternal Igf $2 r$ expression in undifferentiated and differentiated wild-type and targeted ES cells. Numbers are ratios of maternal (S12) to paternal $(+)$ allele. The ratio at day 0 in $\mathrm{S} 12 /+$ cells was set to 1 (asterisk) and an increased ratio at day 5 indicates higher expression of the maternal relative to the paternal allele. The ratios of all targeted ES cell clones (S12/APD, S12/APD-PGK and S12/APD-TET) at day 5 are not significantly different from control cells $(\mathrm{S} 12 /+)$. Error bars represent standard deviation of three technical replicates (one biological replica). (B) As (A), but cells were cultured at day 5 for $10 \mathrm{~h}$ with or without $(+/-)$ emetine. Mean values and standard deviations of three biological replicates are shown. ${ }^{*}$ Set to 17.5 , the value obtained from day $5 \mathrm{~S} 12 /+$ cells shown in (A). A Student's $t$-test (two-tailed, equal variance) shows that S12/APD and S12/APD-TET cells show a significant lower ratio of maternal to paternal Igf2r under emetine treatment compared with S12/ + cells, whereas S12/APD-PGK cells do not $(P>0.1)$. (C) QPCR showing changes in Airn and $H 19$ expression after emetine treatment in targeted ES cells differentiated for 5 days. Mean values and standard deviations of 6 (Airn) or 15 (H19) biological replicates are shown. *Set to 100

emetine-treated wild-type $\mathrm{S} 12 /+$ differentiated cells (that show Igf2 $r$ imprinted expression) is $12: 1$. A similar maternal/paternal ratio was obtained from emetine-treated S12/ APD-PGK differentiated cells. This indicates that a PGKdriven Airn transcript is able to specifically repress the paternal Igf $2 r$ promoter in agreement with results obtained above from RNA FISH and DNA methylation analyses. 
In contrast, the maternal/paternal ratio from emetine-treated S12/APD and non-induced S12/APD-TET differentiated cells is significantly lowered, to approximately $4: 1 \quad(P<0.002$; Student's $t$-test). This indicates emetine treatment stabilized the paternal APD-Igf $2 r$ allele and that these cells express paternal and maternal $I g f 2 r$, in agreement with data obtained above from RNA FISH and DNA methylation analyses. The maternal/paternal ratio does not reach 1:1 in S12/APD and S12/APD-TET ES cells, most likely due to incomplete NMD inhibition by emetine. However, the ratio is significantly lower than in S12/ + and S12/APD-PGK cells (Figure 6B). Thus the allele-specific QPCR assay shows that the APD-PGK allele represses the paternal Igf2r promoter.

The maternal/paternal ratio in emetine-treated wild-type S12/ + differentiated cells was 33\% lower than that observed in untreated S12/ + cells (12:1 compared with 19:1). This reduction could be explained by a reduction in Airn expression upon emetine treatment and was of potential interest because of a previous report that the NMD pathway stabilizes expression of some spliced ncRNAs such as Xist and the imprinted H19 ncRNA (Ciaudo et al, 2006). We used QPCR to quantify Airn and H19 ncRNA expression in all samples following emetine treatment. Figure 6C shows that Airn expression is reduced by $25-50 \%$ after emetine treatment; however, the H19 ncRNA shows an increase of $25-50 \%$. These results indicate that any stabilization effects by the NMD pathway are not translation dependent, and perhaps shows that factors needed for full expression or turnover of ncRNAs are short-lived proteins and lost after emetine treatment.

\section{Transcription is required to protect the paternal Airn CpG island from DNA methylation}

The Airn promoter is located just upstream of a CpG island that is methylated during oocyte maturation on the maternal chromosome, whereas the paternal copy lacks methylation (Seidl et al, 2006). To test whether the absence of paternal Airn CpG island methylation is a passive process or requires Airn transcription, we assayed the methylation status of the paternal Airn CpG island in ES cells carrying an APD or exogenous Airn promoter replacement on the paternal allele. Figure 7 shows that ES cells with an active strong exogenous Airn promoter (S12/APD-PGK) are unmethylated at an SfuI site that is diagnostic for the methylation status of the paternal Airn CpG island (Stoger et al, 1993). In contrast, ES cells lacking the Airn promoter (APD) or with a lowly expressed Airn promoter (non-induced APD-TET) gain paternal Airn CpG island methylation in undifferentiated ES cells, which increases slightly during differentiation. DNA methylation on the paternal Airn CpG island in differentiated ES cells was similar in induced and non-induced APD-TETRolo cells (Figure 7), indicating that the presence of an activated rtTA transactivator does not change established methylation patterns. Taken together, this indicates that the unmethylated status of the paternal Airn CpG island is an active process requiring transcriptional run through from a strong upstream promoter.

\section{Discussion}

We show, by deletion of the Airn ncRNA promoter in ES cells and its replacement with a strong PGK promoter or a

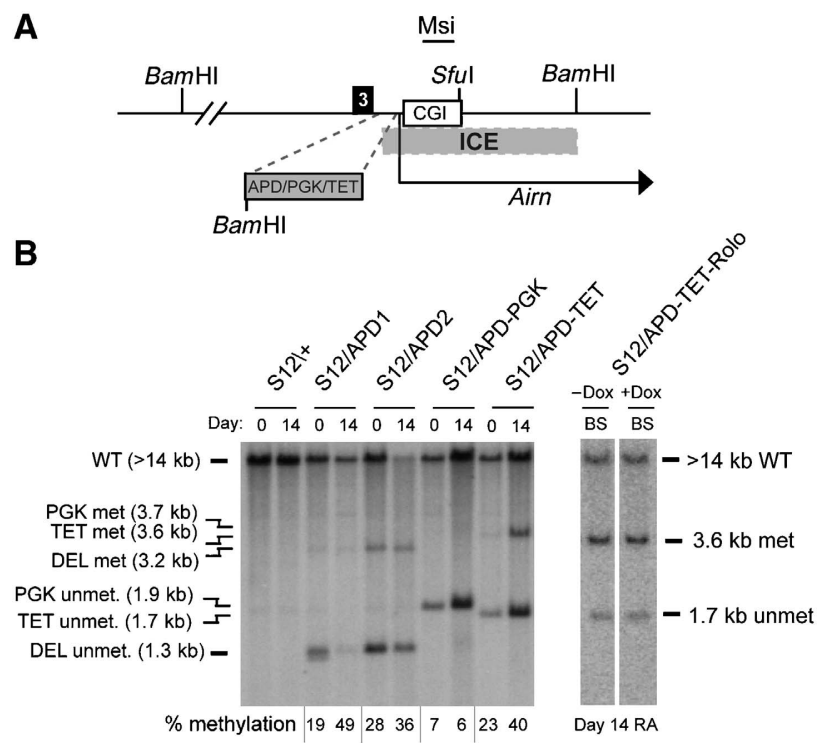

Figure 7 Airn expression protects the Airn CpG island from DNA methylation. (A) Map of a $20-\mathrm{kb}$ region containing the Airn promoter. White box labelled CGI: CpG island; dark grey box: Airn promoter deletion or replacement cassette, which introduced a BamHI site. ICE, details in Figure 1A, Msi Southern blot probe, SfuI: methyl-sensitive site assayed in (B). (B) Left panel: Southern blot on genomic DNA of days 0 and 14 RA differentiated ES cells digested with BamHI $+S f u I$ and probed with fragment Msi. Wildtype fragments from $\mathrm{S} 12 /+$ are too large to separate and migrate together above $14 \mathrm{~kb}$, all fragments below this arise from the paternally targeted allele. In S12/APD and S12/APD-TET cells, no or weak Airn transcription leads to gain of DNA methylation on the paternal Airn CpG island (met). DNA methylation is prominent in differentiated ES cells at day 14 (36-49\% methylation); however, some DNA methylation is also present in undifferentiated ES cells (19-28\% methylation). In contrast, S12/APD-PGK that shows strong Airn expression lacks DNA methylation on the Airn CpG island (unmet). Numbers below indicate the relative intensities of methylated bands (ImageJ). Right panel: the same methylation assay performed on S12/APD-TET-Rolo cells differentiated for 14 days minus $(-)$ or plus $(+)$ doxycycline (Dox), shows that in both conditions the Airn $\mathrm{CpG}$ island gains similar levels of DNA methylation.

non-induced or induced TET promoter, that all functional regions regulating the transcriptional features and the silencing properties of the Airn ncRNA are located within its $108 \mathrm{~kb}$ transcribed gene body. Although transcription of Airn above a critical level is necessary for silencing and DNA methylation of the paternal Igf2r promoter, we also demonstrate a role for Airn transcription in protecting its own CpG island from de novo DNA methylation.

\section{The APD deletion contains essential parts of the Airn promoter}

Deletion of $959 \mathrm{bp}$ upstream of the Airn ncRNA main transcriptional start site on the paternal chromosome led to loss of all Airn transcripts, including all Airn splice variants. This shows that an essential part of the Airn promoter in this region controls both unspliced and spliced Airn transcription. It is notable that the Airn promoter, defined in this and in a previous transient transfection study (Lyle et al, 2000), is immediately upstream of a $\mathrm{CpG}$ island that contains a paternal-specific DNase1 hypersensitive site and 13 predicted GC boxes and 12 consensus sites for Myc-associated zinc fingers (http://www.genomatix.de). Thus, the Airn CpG island is not 
part of the promoter lying upstream to the transcription start, although it may contribute in an as yet undefined manner to regulate Airn transcription initiation or elongation. Earlier reports that have mapped promoters to be inside the $5^{\prime}$ part of $\mathrm{CpG}$ islands have considered the $\mathrm{CpG}$ island to be part of the promoter; however, its specific role in initiation or elongation of transcription has not been studied (Antequera, 2003).

\section{NMD of Igf2r in differentiated ES cells carrying the APD allele}

The 959 bp APD ended 3 bp upstream of the $3^{\prime}$ splice acceptor site of Igf $2 r$ exon 3. In differentiated ES cells, this deletion induced mis-splicing of Igf $2 r$ such that exon 2 spliced to exon 4 and the resultant transcript that contained multiple premature stop codons was not detected in stable mRNA populations. The stabilization of the mis-spliced Igf $2 r$ transcript by translation inhibitors such as emetine indicates but does not prove a role for the NMD pathway (Behm-Ansmant et al, 2007). It is possible that Igf2r exon 2-3 splicing elements such as a pyrimidine tract, a branch site or an intronic splice enhancer (Kim et al, 2008; Seth et al, 2008) are located in the 959 bp Airn promoter region. Notably in undifferentiated APD ES cells, paternal Igf $2 r$ mRNA accumulation was not affected. The NMD pathway is active in undifferentiated ES cells (Shigeoka et al, 2005), this could indicate that missplicing is a feature of high levels of gene expression as differentiated cells express 15- to 20-fold more Igf2r than undifferentiated cells (Figure 6). The transcriptional elongation rate is thought to be a crucial regulator of alternative and mis-splicing events (Hicks et al, 2006; Lavelle, 2007). Therefore, it is possible that low Igf2r expression in undifferentiated cells favours correct splicing events through slow elongation rates, whereas upregulation favours exon skipping.

\section{Airn expression protects its own CpG island from DNA methylation}

Although the DNA methylation mark on the maternal ICE that contains most of the Airn promoter and the CpG island is set in oocytes, the paternal ICE stays unmethylated in spermatogenesis and throughout development. It was suggested that short sequences inside a $113 \mathrm{bp}$ 'imprinting box' in the Airn CpG island protect the paternal allele from de novo methylation (Birger et al, 1999). However, these sequences are retained in all APD and replacement alleles studied here and they did not protect the paternal ICE from de novo methylation in ES cells. Instead, our analysis indicates that the methylation-free state of the paternal ICE is maintained by active transcription from the upstream Airn promoter. Moreover, only Airn expression from the endogenous promoter or from the strong PGK replacement promoter, but not weak expression from the non-induced TET promoter could maintain the Airn CpG island in a methylation-free state, indicating that a certain expression threshold level is required. Interestingly, high expression of the TET transactivator during ES cell differentiation failed to reverse the DNA methylation of the APD-TET Airn CpG island, indicating that once the methylation mark is gained it is not reversed by high levels of the activated rtTA transactivator. A protective role for transcription against DNA methylation is in agreement with current suggestions that DNA methylation may not silence active promoters, but affects genes already silenced (Bird, 2002). It is also in agreement with results from diverse areas of research. For example, experiments deleting transcription factor-binding sites induce DNA methylation on CpG islands (Brandeis et al, 1994; Macleod et al, 1994), and the de novo methyltransferase-regulating factor DNMT3L cannot bind histone $\mathrm{H} 3$ modified by $\mathrm{K} 4$ trimethylation, a mark for expressed promoters (Ooi et al, 2007). It is surprising that undifferentiated ES cells without detectable Airn transcripts gain DNA methylation on the Airn CpG island only when a strong promoter is lacking. However, it was recently found that short initiating transcripts are found on promoters in undifferentiated human ES cells even when the associated gene is not expressed. These promoters contained H3K4me3 and bound the initiating form of RNAPII, but the gene did not show H3K36me3 or full-length transcripts (Guenther et al, 2007). This might represent a genome-wide transcription mechanism to protect $\mathrm{CpG}$ island promoters from de novo methylation. Interestingly, $\mathrm{H} 3 \mathrm{~K} 4 \mathrm{me} 3$ is found on the Airn promoter in undifferentiated ES cells that lack expression of Airn (http://www.broad.mit.edu/ seq_platform/chip/).

\section{The Airn promoter does not suppress Airn ncRNA splicing}

The Airn macro ncRNA differs from mammalian RNAPII mRNA transcripts in transcriptional and post-transcriptional features. Unspliced Airn is relatively unstable and nuclear localized, and its termination appears imprecise as two widely spaced 3 ' polyadenylation sites, have been identified (Seidl et al, 2006). However, its most remarkable feature is that $95 \%$ of Airn ncRNA transcripts are unspliced despite in silico prediction of multiple splice sites throughout its $108 \mathrm{~kb}$ length (http://www.fruitfly.org/cgi-bin/seq_tools/ splice.html). This indicates that splicing is actively suppressed. Splicing suppression could occur at the level of the promoter or arise from specific splicing silencer sequences (Kornblihtt, 2005, 2006; Wang et al, 2006). The data presented here exclude a role for the endogenous promoter, as PGK-driven Airn transcripts remain mainly unspliced. However, both the PGK and TET promoters slightly favoured splicing to downstream exons, which led to moderate downregulation of splice variants 1 and $1 \mathrm{a}$, and upregulation of splice variants 2 and 3 . Although this may indicate a role for the Airn promoter in modulating alternative splicing events, it excludes a role for the promoter in determining the unspliced to spliced ratio.

\section{The silencing activity of the Airn ncRNA depends on a critical expression level}

We show here that a PGK promoter can produce a functional Airn transcript with silencing activity that abolishes Igf $2 r$ transcription and induces gain of DNA methylation in differentiated ES cells. In contrast, the weakly expressed noninduced TET promoter lacks the ability to silence $\operatorname{Igf} 2 r$ in cis. However, induction of high Airn ncRNA expression from the TET promoter is able to repress Igf $2 r$, albeit in an incomplete manner due to the gain of methylation on the Airn CpG island in the APD-TET allele. Thus, the silencing activity of Airn in differentiated ES cells depends not just on an active promoter expressing Airn but on a critical expression level. Airn is normally not expressed in undifferentiated 
ES cells that consequently show biallelic Igf2r expression (Wang et al, 1994; Braidotti et al, 2004). However, the PGK promoter used here is a ubiquitously expressed promoter and PGK-Airn was expressed in undifferentiated ES cells to approximately $50 \%$ of the level seen in differentiated ES cells (Figure 2). This led to a low level of DNA methylation on the Igf $2 r$ promoter, which was not obviously correlated with Igf $2 r$ repression as measured by the allele-specific QPCR assay (Figures 4 and 6). This may indicate this assay lacks the sensitivity to detect a small difference in expression between the maternal and paternal Igf2 $r$ alleles (that are expressed at relatively low levels in undifferentiated ES cells) or that Airn cannot induce silencing in undifferentiated ES cells. This question will be further investigated by generating Airn alleles with stronger expression in undifferentiated ES cells.

\section{Models of Airn-mediated gene silencing}

One of the most intriguing aspects of Airn-dependent gene silencing is the ability of Airn transcription to silence neighbouring genes in cis, although its own promoter is unaffected. In these studies, a mouse PGK promoter is able to silence Igf2 $r$ in differentiated ES cells, without being itself affected. Currently, two models have been suggested to explain the silencing activity of the Airn ncRNA. The RNA-directed targeting model is based on parallels to $\mathrm{X}$ chromosome inactivation and proposes a function for the Airn ncRNA itself. This model proposes that the Airn ncRNA coats the silenced region and recruits effector proteins that induce widespread repressive epigenetic modifications (Pauler et al, 2007). Intuitively, this model implies a special ability of the Airn promoter to resist silencing as the induced epigenetic changes on the paternal allele silence $\operatorname{Ig} f 2 r$, Slc22a2 and Slc22a3, but not the Airn promoter. Our findings argue against this model, as the PGK promoter in the APDPGK allele was not affected by Airn transcription. The PGK promoter used here comes from the X-linked Pgk1 gene that is known to be susceptible to X chromosome inactivation and DNA methylation, so it is not intrinsically resistant to epigenetic silencing (Pfeifer et al, 1989). A further argument against an RNA-directed targeting model operating in embryonic cells comes from analysis of parental-specific histone modifications, which found that repressive histone modifications that are dependent on Airn ncRNA expression do not spread throughout the silenced region, but instead are restricted to the silent Igf2r promoter and to one pseudogene element inside the Airn gene body (Regha et al, 2007).

The second silencing model called the transcriptional interference model proposes that the ncRNA itself is not necessary, instead the function of Airn ncRNA transcription is sufficient because it blocks the interaction of effector proteins to transcriptional activators within the Airn gene body (Seidl et al, 2006). Expression of large numbers of mammalian genes has been shown to be controlled by transcriptional interference (Shearwin et al, 2005; Petruk et al, 2006; Abarrategui and Krangel, 2007; Racanelli et al, 2008). In all these examples, a non-coding RNA overlaps the silenced gene promoter. In the Igf $2 r$ imprinted gene cluster, Airn silences three genes in cis but only overlaps Igf $2 r$. The other two silenced genes Slc22a2 and Slc22a3 lie more than $80 \mathrm{~kb}$ upstream to Airn and lack any sequence homology (these genes show only imprinted expression in placental trophoblast and so could not be assayed in ES cells that cannot differentiate into placental cells). However, a transcriptional interference model can explain silencing of nonoverlapped genes if transcription interferes with a common regulatory element (Seidl et al, 2006). Our studies are in agreement with a transcriptional interference model operating at the Igf $2 r$ imprinted cluster. Transcription interference has been shown to depend on the promoter strength of an overlapping transcription pair (Shearwin et al, 2005). Hence, it is feasible that the strongly expressed PGK promoter and the induced TET promoter, but not the weakly expressed noninduced TET promoter, are able to functionally replace the Airn promoter in $\operatorname{Igf} 2 r$ silencing. Taken together, the results presented here exclude a role for the Airn promoter in the silencing process, but highlight the importance of the Airn transcriptional unit and thus further support the transcriptional interference model of $\operatorname{Ig} f 2 r$ silencing.

\section{Materials and methods}

\section{Construction of targeting plasmids}

The homology region of $7.6 \mathrm{~kb}$ for all targeting vectors was constructed by joining restriction fragments with tailored PCR products leading to homology fragments reaching from XhoI to the splice acceptor of Igf2r exon 3 and from Airn transcription start T1 to the next KpnI site. A loxP511-flanked hygromycin and thymidine kinase cassette was used for selection. In the APD construct, the selection cassette replaced $959 \mathrm{bp}$ containing the Airn promoter (126236-127195 bp; AJ249895). For APD-PGK, a 518-bp fragment of the PGK promoter was cut out with EcoRI and XhoI from pPGKHygro (gift from A Wutz), for APD-TET the $438 \mathrm{bp}$ tetOP promoter from pTET-Splice (Invitrogen) was cut with XhoI and SacII, and ligated to the selection cassette. Electroporation and hygromycin selection were performed using standard conditions. Cassette removal was performed by electroporation of the pMC-Cre plasmid. All targeted alleles were sequenced from PCR of genomic DNA using primers: F: TGGCAGCCCATAGTGGTGTTGA and R: CTCGCA TTGCCGCGCTTCAC. PCR fragments were cloned into pGEM-T Easy (Promega). Two clones from each allele were sequenced from both ends (Supplementary Figure 1).

\section{Generation of APD-TET ES cells carrying a transactivator gene targeted into the ROSA26 locus}

APD-TET ES cells with a tetracycline-dependent transcriptional activator under the control of the ROSA26 promoter were generated by introducing the ClaI linearized M2rtTA construct (Beard et al, 2006) into APD-TET cells. Screening for homologous recombination was performed by DNA blotting of EcoRV-digested DNA and a PCR-amplified probe (5'-GCACCGGCCAATAAGTGT- ${ }^{\prime}$, 5'-GTAGGCAATACCCAGGCAAA- $3^{\prime}$ ). Single integration and the integrity of the recombined allele were checked on the same DNA blot with a $0.7 \mathrm{~kb} E c o R I-B a m H I$ fragment (from the M2rtTA construct) as a probe.

\section{ES cell culture and differentiation assays}

ES cell lines were grown in standard culture conditions on irradiated primary embryonic fibroblast feeders with the IPdel/ Thp genotype (maternal Igf $2 r$ promoter deletion/paternal Thp deletion; Sleutels et al, 2003) that lack maternal and paternal copies of Igf2r and carry a repressed DNA methylated maternal Airn promoter. Differentiation was induced by withdrawal of LIF, depletion of feeder cells and addition of $0.08 \mu \mathrm{g} / \mathrm{ml}$ all trans RA (Sigma). The tetracycline-responsive activator was induced by the presence of $1 \mu \mathrm{g} / \mathrm{ml}$ doxycycline (Sigma) continuously for the differentiation period

\section{RNA and DNA analyses}

For translation inhibition, ES cells were differentiated with RA for 5 days and emetine hydrochloride hydrate $(100 \mu \mathrm{g} / \mathrm{ml}$; Sigma) was added for $10 \mathrm{~h}$. RNA was treated with DNaseI before reverse transcription. RNA FISH was performed using standard protocols and strand-specific intronic Igf2r probes FP1 (AIFP1F 5'-GCTGGTCCTTACCTTGTGGA-3'; AIFP1R 5'-GCAAGACCACATCA 
CACACC- $3^{\prime}$ ) and FP3 (AIFP3F 5'-TCCTCAGGTACCATGCTATGC-3'; AIFP3R 5'-GGCAGGTTCTCTTGTTGAGG-3'). Fluorescent spots were counted by two people and one count was performed blind. $5^{\prime}$ RACE was performed with the FirstChoiceRLM-RAC (Ambion) and primers $5^{\prime}$-GCTCTAAATCGCCCGTAAAC- $3^{\prime}$ and $5^{\prime}$-TTCACCCTAGCG CTGAATCT- $3^{\prime}$. Real-time QPCR and conventional PCR used the following primers not described earlier (Seidl et al, 2006): Igf2rex1: 5'-GCCGTTCAGCTGGGACC-3'; Igf2rex4: 5'-GGCTGCAG TCCTCCATT-3'. Igf2r allele-specific PCR assay (MutSEF: 5'CTGGCCTTCCCCTCCTGT-3'; WtSEF: 5'-TGGCCTTCCCCTCCTGC-3', GESER2: 5'-GCTATGACCTGTCTGTGTTGGCT-3'). DNA methylation probes Msi (AJ249895: 124993-126087 bp), Psi (AJ249895: 124370124992 bp), OT2.4 (AJ249895: 120967-123159 bp), Be2i (AJ249895: 97091-99081 bp).

\section{References}

Abarrategui I, Krangel MS (2007) Noncoding transcription controls downstream promoters to regulate T-cell receptor alpha recombination. EMBO J 26: 4380-4390

Antequera F (2003) Structure, function and evolution of CpG island promoters. Cell Mol Life Sci 60: 1647-1658

Beard C, Hochedlinger K, Plath K, Wutz A, Jaenisch R (2006) Efficient method to generate single-copy transgenic mice by sitespecific integration in embryonic stem cells. Genesis 44: 23-28

Behm-Ansmant I, Kashima I, Rehwinkel J, Sauliere J, Wittkopp N, Izaurralde E (2007) mRNA quality control: an ancient machinery recognizes and degrades mRNAs with nonsense codons. FEBS Lett 581: 2845-2853

Bentley DL (2005) Rules of engagement: co-transcriptional recruitment of pre-mRNA processing factors. Curr Opin Cell Biol 17: 251-256

Bird A (2002) DNA methylation patterns and epigenetic memory. Genes Dev 16: 6-21

Birger Y, Shemer R, Perk J, Razin A (1999) The imprinting box of the mouse Igf2r gene. Nature 397: 84-88

Braidotti G, Baubec T, Pauler F, Seidl C, Smrzka O, Stricker S, Yotova I, Barlow DP (2004) The Air noncoding RNA: an imprinted cissilencing transcript. Cold Spring Harb Symp Quant Biol 69: 55-66

Brandeis M, Frank D, Keshet I, Siegfried Z, Mendelsohn M, Nemes A, Temper V, Razin A, Cedar H (1994) Sp1 elements protect a CpG island from de novo methylation. Nature 371: 435-438

Carninci P, Kasukawa T, Katayama S, Gough J, Frith MC, Maeda N, Oyama R, Ravasi T, Lenhard B, Wells C, Kodzius R, Shimokawa $\mathrm{K}$, Bajic VB, Brenner SE, Batalov S, Forrest AR, Zavolan M, Davis MJ, Wilming LG, Aidinis V et al (2005) The transcriptional landscape of the mammalian genome. Science 309: 1559-1563

Cheng J, Kapranov P, Drenkow J, Dike S, Brubaker S, Patel S, Long J, Stern D, Tammana H, Helt G, Sementchenko V, Piccolboni A, Bekiranov S, Bailey DK, Ganesh M, Ghosh S, Bell I, Gerhard DS, Gingeras TR (2005) Transcriptional maps of 10 human chromosomes at 5-nucleotide resolution. Science 308: 1149-1154

Ciaudo C, Bourdet A, Cohen-Tannoudji M, Dietz HC, Rougeulle C, Avner P (2006) Nuclear mRNA degradation pathway(s) are implicated in Xist regulation and $\mathrm{X}$ chromosome inactivation. PLoS Genet 2: e94

Custodio N, Carmo-Fonseca M, Geraghty F, Pereira HS, Grosveld F, Antoniou M (1999) Inefficient processing impairs release of RNA from the site of transcription. EMBO J 18: 2855-2866

Guenther MG, Levine SS, Boyer LA, Jaenisch R, Young RA (2007) A chromatin landmark and transcription initiation at most promoters in human cells. Cell 130: 77-88

Hicks MJ, Yang CR, Kotlajich MV, Hertel KJ (2006) Linking splicing to Pol II transcription stabilizes pre-mRNAs and influences splicing patterns. PLoS Biol 4: e147

Kapranov P, Willingham AT, Gingeras TR (2007) Genome-wide transcription and the implications for genomic organization. Nat Rev Genet 8: 413-423

Kim E, Goren A, Ast G (2008) Alternative splicing: current perspectives. Bioessays 30: 38-47

Kornblihtt AR (2005) Promoter usage and alternative splicing. Curr Opin Cell Biol 17: 262-268

Kornblihtt AR (2006) Chromatin, transcript elongation and alternative splicing. Nat Struct Mol Biol 13: 5-7

Kornblihtt AR, de la Mata M, Fededa JP, Munoz MJ, Nogues G (2004) Multiple links between transcription and splicing. RNA 10: 1489-1498

\section{Supplementary data}

Supplementary data are available at The EMBO Journal Online (http://www.embojournal.org).

\section{Acknowledgements}

This study was supported by FWF SFB F17 Modulators of RNA Fate and Function (SFBF01718 B10), the EU FW6 Integrated Project 'HEROIC' Highthroughput Epigenetic Regulatory Organization in Chromatin (LSHG-CT-2005-018883) and the NoE 'The Epigenome' (LSHG-CT-2004-053433). We thank Erwin Wagner, Anton Wutz for materials and help with ES culture, and Sophia Blake, Steven Pollard and all the Barlow Group for advice and support.

Lavelle C (2007) Transcription elongation through a chromatin template. Biochimie 89: 516-527

Li E (2002) Chromatin modification and epigenetic reprogramming in mammalian development. Nat Rev Genet 3: 662-673

Lyle R, Watanabe D, te Vruchte D, Lerchner W, Smrzka OW, Wutz A, Schageman J, Hahner L, Davies C, Barlow DP (2000) The imprinted antisense RNA at the Igf2r locus overlaps but does not imprint Mas1. Nat Genet 25: 19-21

Macleod D, Charlton J, Mullins J, Bird AP (1994) Sp1 sites in the mouse aprt gene promoter are required to prevent methylation of the CpG island. Genes Dev 8: 2282-2292

Mancini-Dinardo D, Steele SJ, Levorse JM, Ingram RS, Tilghman SM (2006) Elongation of the Kcnq1ot1 transcript is required for genomic imprinting of neighboring genes. Genes Dev 20: $1268-1282$

Mattick JS (2005) The functional genomics of noncoding RNA. Science 309: 1527-1528

Ooi SK, Qiu C, Bernstein E, Li K, Jia D, Yang Z, Erdjument-Bromage H, Tempst P, Lin SP, Allis CD, Cheng X, Bestor TH (2007) DNMT3L connects unmethylated lysine 4 of histone $\mathrm{H} 3$ to de novo methylation of DNA. Nature 448: 714-717

Pauler FM, Koerner MV, Barlow DP (2007) Silencing by imprinted noncoding RNAs: is transcription the answer? Trends Genet 23: 284-292

Penny GD, Kay GF, Sheardown SA, Rastan S, Brockdorff N (1996) Requirement for Xist in X chromosome inactivation. Nature 379: 131-137

Petruk S, Sedkov Y, Riley KM, Hodgson J, Schweisguth F, Hirose S, Jaynes JB, Brock HW, Mazo A (2006) Transcription of bxd noncoding RNAs promoted by trithorax represses Ubx in cis by transcriptional interference. Cell 127: 1209-1221

Pfeifer GP, Steigerwald SD, Mueller PR, Wold B, Riggs AD (1989) Genomic sequencing and methylation analysis by ligation mediated PCR. Science 246: 810-813

Racanelli AC, Turner FB, Xie LY, Taylor SM, Moran RG (2008) A mouse gene that coordinates epigenetic controls and transcriptional interference to achieve tissue-specific expression. Mol Cell Biol 28: 836-848

Regha K, Sloane MA, Huang R, Pauler FM, Warczok KE, Melikant B, Radolf M, Martens JH, Schotta G, Jenuwein T, Barlow DP (2007) Active and repressive chromatin are interspersed without spreading in an imprinted gene cluster in the mammalian genome. Mol Cell 27: 353-366

Seidl CI, Stricker SH, Barlow DP (2006) The imprinted Air ncRNA is an atypical RNAPII transcript that evades splicing and escapes nuclear export. $Е M B O J$ 25: 3565-3575

Seth P, Miller HB, Lasda EL, Pearson JL, Garcia-Blanco MA (2008) Identification of an intronic splicing enhancer essential for the inclusion of FGFR2 exon IIIc. J Biol Chem 283: 10058-10067

Shearwin KE, Callen BP, Egan JB (2005) Transcriptional interference-a crash course. Trends Genet 21: 339-345

Shigeoka T, Kawaichi M, Ishida Y (2005) Suppression of nonsense-mediated mRNA decay permits unbiased gene trapping in mouse embryonic stem cells. Nucleic Acids Res 33: e20

Sleutels F, Tjon G, Ludwig T, Barlow DP (2003) Imprinted silencing of Slc22a2 and Slc22a3 does not need transcriptional overlap between Igf2r and Air. EMBO J 22: 3696-3704 
Sleutels F, Zwart R, Barlow DP (2002) The non-coding Air RNA is required for silencing autosomal imprinted genes. Nature 415: 810-813

Stoger R, Kubicka P, Liu CG, Kafri T, Razin A, Cedar H, Barlow DP (1993) Maternal-specific methylation of the imprinted mouse Igf2r locus identifies the expressed locus as carrying the imprinting signal. Cell 73: 61-71

Wang Z, Xiao X, Van Nostrand E, Burge CB (2006) General and specific functions of exonic splicing silencers in splicing control. Mol Cell 23: 61-70
Wang ZQ, Fung MR, Barlow DP, Wagner EF (1994) Regulation of embryonic growth and lysosomal targeting by the imprinted Igf2/Mpr gene. Nature 372: 464-467

Wutz A, Theussl HC, Dausman J, Jaenisch R, Barlow DP, Wagner EF (2001) Non-imprinted Igf2r expression decreases growth and rescues the Tme mutation in mice. Development 128: $1881-1887$

Yasuda J, Hayashizaki Y (2008) The RNA continent. Adv Cancer Res 99: $77-112$ 